\title{
Does the efficacy of epidermal growth factor receptor (EGFR) tyrosine kinase inhibitor differ according to the type of EGFR mutation in non-small cell lung cancer?
}

Yong Won Choi and Jin-Hyuk Choi

Department of HematologyOncology, Ajou University School of Medicine, Suwon, Korea

Received: July 13, 2016

Accepted: March 2, 2017

\section{Correspondence to}

Jin-Hyuk Choi, M.D.

Department of Hematology-Oncology, Ajou University School of Medicine, 164 World cup-ro, Yeongtong-gu, Suwon 16499, Korea

Tel: +82-31-219-4920

Fax: $+82-31-219-5983$

E-mail:jhchoimd@ajou.ac.kr
An exon 19 deletion and a L858R mutation in exon 21 of the epidermal growth factor receptor (EGFR) are the two most common mutations that predict favorable efficacy of EGFR tyrosine kinase inhibitors (TKIs) in patients with non-small cell lung cancer (NSCLC). Many retrospective and prospective studies, as well as meta-analyses including patients with NSCLC with various lines of EGFR TKI treatment, have demonstrated longer progression-free survival and sometimes more favorable overall survival in patients with an exon 19 deletion than those with the L858R or other mutations. In contrast, some clinical studies, including phase III trials, have demonstrated no difference in the efficacy of EGFR TKIs according to the EGFR mutation type. Therefore, the existence of clinically significant differences in sensitivity to EGFR-TKIs among different EGFR mutation subtypes remains controversial. In this review, we summarize the evidence suggesting different outcomes according to the type of EGFR mutation in patients with advanced NSCLC who were treated with EGFR-TKIs, along with their clinical significance. We also discuss possible mechanisms that can explain the different sensitivities to EGFR TKIs between cases with an exon 19 deletion and those with the L858R mutation.

Keywords: Carcinoma, non-small-cell lung; Receptor, epidermal growth factor; Tyrosine kinase inhibitor; Exon 19 deletion

\section{INTRODUCTION}

Non-small cell lung cancer (NSCLC), which comprises approximately $87 \%$ of newly diagnosed lung cancers, is the most common malignancy and the leading cause of cancer-related death in many countries, including Korea $[1,2]$. About $70 \%$ of patients with NSCLC are diagnosed with advanced disease [3,4]. Despite advances in palliative chemotherapy, the prognosis of patients with advanced NSCLC remains very poor, with a median sur- vival of 8 to 12 months [4-6]. However, since epidermal growth factor receptor (EGFR) tyrosine kinase inhibitors (TKIs) have become standard practice as first-line palliative chemotherapy in patients with EGFR mutation-positive advanced NSCLC [7], based on the discovery that EGFR activating mutations are closely related to high sensitivity to EGFR-TKIs [8-10], patients treated with EGFR-TKIs show significantly improved clinical outcomes compared with those who receive standard cytotoxic chemotherapy [11-13]. This finding has strong 
clinical implications, particularly in Korea, where a recent nationwide survey demonstrated EGFR mutations in $34.3 \%$ of 1,753 patients with NSCLC [14].

The existence of a clinically meaningful difference in the sensitivity to EGFR TKIs among several types of EGFR activating mutations is controversial. For example, an early tumor xenograft study compared EGFR TKI sensitivity between the two most common types of EGFR mutations and showed higher sensitivity to erlotinib in tumor cells harboring the exon 19 deletion than in those harboring the L858R mutation [15]. Several clinical studies on EGFR-TKI treatment demonstrated longer progression-free survival (PFS) and/or overall survival (OS) in patients with the exon 19 deletion than those with the L858R mutation [16-22]. However, in other studies, including phase III trials, no significant differences were noted in the efficacy of EGFR TKIs for different types of EGFR mutations [11,12,23-26]. In this review, we summarize the evidence that supports differences in outcomes according to the type of EGFR mutation in patients with NSCLC who received EGFR-TKI treatment. We also discuss the clinical relevance of these differences, as well as in vitro experimental data that point to possible mechanisms to explain the different sensitivities of the exon 19 deletion and L858R mutation to EGFR-TKIs.

\section{COMPARISON OF CLINICAL OUTCOMES IN PATIENTS WITH THE EGFR EXON 19 DELETION AND L858R MUTATION FOLLOWING EGFR TKI THERAPY}

Many studies have analyzed clinical differences between the EGFR exon 19 deletion and the L858R mutation in patients with advanced NSCLC treated with EGFR TKIs. In 2006, Riely et al. [17] and Jackman et al. [16] separately reported that median PFS (12 months vs. 5 months, $p=0.01 ; 24$ months vs. 10 months, $p=0.04)$ and OS (34 months vs. 8 months, $p=0.01$; 38 months vs. 17 months, $p=0.04$ ) were significantly longer in patients with the EGFR exon 19 deletion than in those with the L858R mutation following treatment with gefitinib or erlotinib. However, these studies retrospectively analyzed a relatively small number of patients $(<40)$ who had received different lines of these two EGFR-TKIs. In contrast, at the same time, four prospective phase II trials that in- cluded patients treated with first-line gefitinib therapy showed no differences in the response rate (RR) among patients with NSCLC harboring different EGFR mutation subtypes [24-27]. Nonetheless, these clinical trials also included a relatively small numbers of patients with EGFR mutations and did not report PFS or OS according to the EGFR mutation subtype.

In 2009, Rosell et al. [21] reported the first large-scale prospective analysis demonstrating different clinical outcomes between the two activating mutations in patients with NSCLC receiving erlotinib. In that study, 113 of 217 patients with EGFR mutations received erlotinib as first-line therapy. The RR (odds ratio, 3.08; $p=0.001$ ) was significantly higher in patients with the EGFR exon 19 deletion, and the PFS (hazard ratio [HR], 1.92; $p=0.02$ ) and OS (HR, 2.98; $p=0.002$ ) was significantly shorter in patients with the L858R mutation. However, in 2010, two Japanese phase III trials compared first-line gefitinib with standard platinum-based doublet chemotherapy but reported no difference in PFS between patients with the exon 19 deletion and those with the L858R mutation who were treated with gefitinib [11,12]. In contrast, at the same time, two Korean retrospective studies designed to compare the clinical outcomes of patients with these two mutations demonstrated a significantly longer PFS for patients with the exon 19 deletion than those with the L858R mutation following treatment with gefitinib or erlotinib as first- or more lines of therapy $[18,19]$.

Several reports support the clinical differences between EGFR mutation subtypes, but no prospective study has been specifically designed to clarify the different clinical efficacy of T'KIs according to EGFR mutation subtype. Instead, four meta-analyses have compared the efficacy of EGFR TKIs among patients with advanced NSCLC and the exon 19 deletion or L858R mutation. In 2014, Wang et al. [28] analyzed 22 studies including 1,082 patients who received EGFR TKIs (gefitinib or erlotinib). PFS ( $p=0.01)$ and OS $(p=0.0001)$ were significantly longer for patients with the exon 19 deletion than those with the L858R mutation. However, that study analyzed data from heterogeneous clinical settings ranging from retrospective studies to prospective clinical trials and included different lines of EGFR TKI therapy. In addition, only three retrospective studies were used in the pooled OS analysis. In contrast, Zhang et al. [29] analyzed 13 studies that included six clinical trials or 
retrospective studies with EGFR-TKIs (e.g., gefitinib, erlotinib, or afatinib) as first-line therapy. An indirect meta-analysis of the six clinical trials revealed a longer PFS in patients with the exon 19 deletion than those with the L858R mutation (HR, 0.59; $p=0.019)$. A direct meta-analysis from another seven retrospective studies revealed a similar result (HR, $0.75 ; p<0.001)$. However, OS data were not reported in this meta-analysis. A meta-analysis by Lee et al. [30] in 2015 analyzed only randomized trials comparing first-line EGFR TKIs with platinum-based combination chemotherapy. Treatment with EGFR TKIs provided a 50\% greater benefit in patients with the exon 19 deletion than those with the L858R substitution in seven trials compared with chemotherapy. A multivariate analysis using individual patient data from the four trials revealed pooled HRs for PFS of 0.26 and 0.44 for the exon 19 deletion and L858R mutation subgroups, respectively, with a significant difference in the interaction analysis after adjusting for smoking status and sex $(p=0.004)$. They did not report the OS of the subgroups because the trials had yet to report mature OS data. The most recent meta-analysis used the largest dataset and included 4,835 patients from 26 trials. The authors reported a significantly longer PFS in patients with the exon 19 deletion than those with the L858R mutation (HR, 0.69; $p<0.001)$ [31].

The available retrospective or prospective studies and meta-analyses appear to support the conclusion that EGFR-TKIs have better efficacy, at least in terms of PFS, in patients with an exon 19 deletion than in those with the L858R mutation, including their use as first-line treatment. However, few reports have demonstrated differences in OS in patients with the exon 19 deletion compared with those with the L858R mutation after first-line EGFR-TKI treatment, although this is considered the standard of care for patients with advanced NSCLC and activating EGFR mutations. In 2011, a Korean phase II trial that included 45 patients with NSCLC treated with first-line gefitinib therapy revealed a significantly longer OS (24 months OS rate: $72.1 \%$ vs. $32.0 \%, p=0.0148$ ) in patients with the exon 19 deletion than those with the L858R mutation. However, no difference in PFS was reported $(p=0.068)$ [22]. Our recent retrospective study analyzing the outcomes of 60 patients with EGFR mutation-positive advanced NSCLC who received first-line gefitinib revealed significantly longer median PFS (20 months vs. 9 months, $p=0.030)$ and $\mathrm{OS}(36$ months vs. 22 months, $p=0.002$ ) in patients with the exon 19 deletion than those with the L858R mutation [32]. In addition, a multivariate analysis showed that the exon 19 deletion was an independent prognostic factor for a favorable $\operatorname{PFS}(p=0.007)$ and OS $(p<0.0001)$.

The most recent meta-analysis showed a significantly greater benefit in terms of $O S$ in patients with the exon 19 deletion than those with the L858R mutation (HR, $0.61 ; p=0.005$ ) [31]. However, the beneficial effect on OS for the exon 19 deletion was only significant in a mixedline setting $(p=0.005)$ and not in a first-line setting $(p=$ 0.156). Furthermore, a combined analysis of two phase III trials (LUX-Lung 3 and LUX-Lung 6) comparing afatinib with cisplatin doublet chemotherapy as a first-line setting in EGFR mutation-positive lung adenocarcinoma (total 709 patients) showed a benefit of afatinib treatment on OS in patients with the exon 19 deletion (median OS: 31.7 months vs. 20.7 months, $p=0.0001$ ) but not those with the L858R mutation(median OS: 22.1 months vs. 26.9 months, $p=0.16$ ) [13]. This result suggests a better OS in patients with the exon 19 deletion than those with the L858R mutation, although OS was not directly compared between the two groups in the afatinib arm [13]. Table 1 summarizes the results of studies that compared the efficacy of first-line EGFR-TKI for advanced NSCLC in terms of PFS and/or OS according to EGFR mutation subtype.

\section{POSSIBLE MECHANISMS TO EXPLAIN THE FA- VORABLE CLINICAL OUTCOMES IN PATIENTS WITH THE EGFR EXON 19 DELETION}

The reason for the observed difference in outcome in patients with the EGFR exon 19 deletion and L858R mutation after treatment with EGFR-TKIs is not clear. The difference in efficacy of EGFR TKIs according to EGFR mutation subtype has been explained by some proposed mechanisms suggested by preclinical studies. Two molecular biology studies comparing the characteristics of wild and mutant EGFRs reported different affinities between TKIs and each EGFR mutation subtype [15,33]. A study by Carey et al. [15] performed an in vitro kinetic analysis of peptide phosphorylation reactions with purified intracellular domains from EGFR wild-type, L858R, 
Table 1. Summary of studies comparing the efficacy of first-line EGFR-TKI for advanced NSCLC according to subtype of EGFR mutation

\begin{tabular}{|c|c|c|c|c|c|c|c|c|c|c|}
\hline \multirow{2}{*}{ Study } & \multirow{2}{*}{$\begin{array}{c}\text { No. of } \\
\text { patients }\end{array}$} & \multirow{2}{*}{$\begin{array}{c}\text { Exon } \\
19\end{array}$} & \multirow{2}{*}{$\mathrm{L} 858 \mathrm{R}$} & \multirow{2}{*}{ TKI } & \multicolumn{3}{|c|}{ Median PFS, mon } & \multicolumn{3}{|c|}{ Median OS, mon } \\
\hline & & & & & Exon 19 & $\mathrm{~L} 858 \mathrm{R}$ & $p$ value & Exon 19 & L858R & $p$ value \\
\hline Maemondo et al. (2010) [11] & 107 & 58 & 49 & G & 11.5 & 10.8 & 0.900 & - & - & - \\
\hline Mitsudomi et al. (2010) [12] & 86 & 50 & 36 & G & 9.0 & 9.6 & 0.681 & - & - & - \\
\hline Kim et al. (2011) [22] & 44 & 29 & 15 & G & 16.5 & 6.9 & 0.068 & NR & 16.2 & 0.015 \\
\hline Choi et al. (2016) [32] & 53 & 28 & 25 & G & 20.0 & 9.0 & 0.030 & 36.0 & 22.0 & 0.002 \\
\hline Yang et al. (2015) [13] & 419 & 236 & 183 & A & - & - & - & 31.7 & 22.1 & - \\
\hline
\end{tabular}

EGFR, epidermal growth factor receptor; TKI, tyrosine kinase inhibitor; NSCLC, non-small cell lung cancer; Exon 19 , exon 19 deletion; PFS, progression-free survival; OS, overall survival; G, gefitinib; NR, not reached; A, afatinib.

and EGFR del746-750. The ratio of the $\mathrm{K}_{\mathrm{i}}$ for erlotinib to the $K_{M}$ for ATP $\left(K_{i} / K_{M}\right)$, which estimates relative binding affinity of erlotinib to the EGFR kinase domain, was 137 and 23 times higher in the EGFR del746-750 subtype compared with that in the EGFR wild-type and L858R subtype, respectively [15]. A study by Mulloy et al. [33] determined the affinity between purified EGFR kinases and gefitinib by equilibrium fluorescence titration. The $\mathrm{K}_{\mathrm{d}}$ values for the wild-type, the L858R mutant, and the del747-753 mutant EGFR were 177.2 $\pm 6.9,38.0 \pm 7.8$, and $13.6 \pm 2.0 \mathrm{nM}$, respectively [33]. These in vitro kinetic assay data indicate a higher affinity of gefitinib and erlotinib for recombinant EGFR with the exon 19 deletion than that with the L858R mutation. However, the clinical relevance of this higher affinity remains to be established.

Some studies have shown differences in EGFR phosphorylation status and its downstream signaling before and after epidermal growth factor (EGF) or TKI treatment in cell lines expressing different EGFR mutations [34,35]. For example, Okabe et al. [35] demonstrated different patterns of EGFR autophosphorylation in the Y1068 residue, a key residue representing activation status of EGFR in NSCLC cell lines, including primary cells from Japanese patients with NSCLC. EGFR autophosphorylation is ligand (EGF)-dependent in H1650 cells harboring the same exon 19 deletion, but is negative for EGFR amplification, whereas Y1068 (but not Y845 and Y1173) is constitutively phosphorylated in PC-9 and Ma-1 cells, which both show EGFR amplification. In contrast, NSCLC cell lines harboring the L858R mutation exhibit ligand (EGF)-independent and constitutive phosphorylation of EGFR at Y845, Y1068, and Y1173, regardless of the absence or presence of EGFR amplification. That study focused on the Y1173 residue due to constitutive phosphorylation in cells with the L858R mutation but not in the exon 19 deletion.

The EGFR Y1173 residue is a major docking site for the adapter protein Shc [36], so Shc phosphorylation was examined in cell lines with these two types of EGFR mutations. A markedly greater basal level of Shc phosphorylation was observed in cells harboring the L858R mutation than in those harboring the exon 19 deletion or wildtype EGFR. These investigators suggested that constitutively active mutant EGFR induces selective activation of downstream effectors as a result of differential patterns of receptor autophosphorylation. That study used several cell lines; however, cell lines established from different patients have genetic and epigenetic heterogeneities even though they have the same EGFR mutation subtype. In addition, EGFR and Shc phosphorylation status in the different EGFR subtypes was not examined in the presence or absence of EGFR-TKIs.

In contrast, Zhu et al. [34] did not use established NSCLC cell lines but generated stable cell lines harboring each of the two mutations with 293 cells that originally did not express EGFR. They confirmed that proliferation of the 293-EGFR del746-750 and 293-EGFR L858R cell lines was similar in the absence of gefitinib, and that proliferation was inhibited more significantly in 293-EGFR del746-750 cells than in 293-EGFR L858R cells in the presence of gefitinib. The baseline level of autophosphorylation in the 293-EGFR del746-750 cells was not different from that in 293-EGFR L858R cells, which agreed with previous studies. However, gefitinib induced a more marked decrease in EGFR autophosphorylation at tyrosine residues 1173,845 , and 1045 and 
a lesser decrease at $\mathrm{Y}_{992}$ in del746-750 cells, compared with the autophosphorylation levels in L858R cells. The phosphorylation levels of Akt and Erk1/2, major downstream signals of EGFR for cell survival and proliferation, decreased more sharply in del746-750 cells than in L858R cells. Taken together, the different phosphotyrosine patterns between these two mutations may be associated with differential response durations of the EGFR TKIs.

In addition to the results of these preclinical studies, it is worth considering that the EGFR exon 19 deletion is not a one-of-a-kind mutation. The most frequent type of exon 19 deletion mutation is del746-750, which comprises $64 \%$ of exon 19 mutations, followed by del747-753InsS, del747-75, del747-750InsP, del746-752InsV, del747-752, and del746-751InsA. Among these, the most common mutation, del746-750, is reported to be slightly more sensitive to EGFR TKIs than the other variants [37,38]. Some discrepancies exist between in vitro studies, but almost all exon 19 deletion mutants are generally more sensitive to EGFR TKIs than that of L858R mutants.

Moreover, a recent meta-analysis of 15 observational studies and three randomized controlled trials revealed that the EGFR-TKI resistant T790M mutation is more frequently encountered in pretreatment samples from patients with the L858R mutation than those with the exon 19 mutation [39]. This result suggests another possible mechanism to explain the better efficacy of EGFR-TKI in deletion 19 patients.

Despite these studies, the mechanism for better efficacy to EGFR TKIs in patients with the exon 19 deletion than those with the L858R mutation remains controversial. Further basic and clinical studies are required to define the exact mechanism that leads to the difference in efficacy in these two common mutations.

\section{CLINICAL RELEVANCE OF THE DIFFERENCES IN EFFICACY OF EGFR-TKIS ACCORDING TO EGFR MUTATION SUBTYPE}

The different outcomes of patients with the EGFR exon 19 deletion versus those with the L858R mutation shown in many studies have several clinical implications. First, EGFR TKIs could be strongly recommended as first-line therapy for patients with advanced NSCLC, particularly those with the EGFR exon 19 deletion. Second, a more precise explanation can be provided to patients and their families regarding the prognosis after EGFR TKI therapy based on the patients' EGFR mutation subtype. Third, these studies suggest that EGFR mutation subtype should be considered a stratification factor in further clinical trials when evaluating the efficacy of EGFR TKIs [32]. Finally, although current guidelines categorize NSCLC with the EGFR exon 19 deletion and L858R mutation as one subtype and suggest the same EGFR TKI treatment, medical oncologists should carefully consider the type of EGFR mutation, not only in treatment decision making but also when designing clinical trials.

\section{CONCLUSIONS}

Several retrospective and prospective studies, as well as meta-analyses, have indicated a general association between the EGFR exon 19 deletion and more favorable outcomes in patients receiving EGFR TKI treatment compared with patients with the L858R mutation, particularly in terms of PFS. Of course, the EGFR mutation subtype alone does not explain the differences in sensitivity to EGFR TKIs. Many other factors, such as EGFR gene copy number, signaling from other ErbB families, and epithelial-to mesenchymal transition status, could contribute to the sensitivity and development of resistance to EGFR TKIs. In addition, further clinical studies are warranted to conclusively define the role of EGFR mutation subtype as a predictive factor in EGFR TKI therapy. Nevertheless, the EGFR mutation subtype should be considered when making treatment decisions or designing new clinical trials for chemotherapy-naïve, EGFR mutation-positive patients with advanced NSCLC.

\section{Conflict of interest}

No potential conflict of interest relevant to this article was reported.

\section{REFERENCES}

1. Jung KW, Won YJ, Kong HJ, Oh CM, Lee DH, Lee JS. Prediction of cancer incidence and mortality in Korea, 2014. 
Cancer Res Treat 2014;46:124-130.

2. Siegel R, Ma J, Zou Z, Jemal A. Cancer statistics, 2014. CA Cancer J Clin 2014;64:9-29.

3. Jett JR, Schild SE, Kesler KA, Kalemkerian GP. Treatment of small cell lung cancer: diagnosis and management of lung cancer, 3rd ed: American College of Chest Physicians evidence-based clinical practice guidelines. Chest 2013;143(5 Suppl):e40oS-e419S.

4. Spira A, Ettinger DS. Multidisciplinary management of lung cancer. N Engl J Med 2004;350:379-392.

5. Schiller JH, Harrington D, Belani CP, et al. Comparison of four chemotherapy regimens for advanced non-smallcell lung cancer. N Engl J Med 2002;346:92-98.

6. Choi YW, Ahn MS, Jeong GS, et al. Is fourth-line chemotherapy routine practice in advanced non-small cell lung cancer? Lung Cancer 2015;87:155-161.

7. Reck M, Popat S, Reinmuth N, et al. Metastatic nonsmall-cell lung cancer (NSCLC): ESMO Clinical Practice Guidelines for diagnosis, treatment and follow-up. Ann Oncol 2014;25 Suppl 3:iii27-iii39.

8. Jang TW, Oak CH, Chang HK, Suo SJ, Jung MH. EGFR and KRAS mutations in patients with adenocarcinoma of the lung. Korean J Intern Med 2009;24:48-54.

9. Lynch TJ, Bell DW, Sordella R, et al. Activating mutations in the epidermal growth factor receptor underlying responsiveness of non-small-cell lung cancer to gefitinib. N Engl J Med 2004;350:2129-2139.

10. Paez JG, Janne PA, Lee JC, et al. EGFR mutations in lung cancer: correlation with clinical response to gefitinib therapy. Science 2004;304:1497-1500.

11. Maemondo M, Inoue A, Kobayashi K, et al. Gefitinib or chemotherapy for non-small-cell lung cancer with mutated EGFR. N Engl J Med 2010;362:2380-2388.

12. Mitsudomi T, Morita S, Yatabe Y, et al. Gefitinib versus cisplatin plus docetaxel in patients with non-small-cell lung cancer harbouring mutations of the epidermal growth factor receptor (WJTOG3405): an open label, randomised phase 3 trial. Lancet Oncol 2010;11:121-128.

13. Yang JC, Wu YL, Schuler M, et al. Afatinib versus cisplatin-based chemotherapy for EGFR mutation-positive lung adenocarcinoma (LUX-Lung 3 and LUX-Lung 6): analysis of overall survival data from two randomised, phase 3 trials. Lancet Oncol 2015;16:141-151.

14. Lee SH, Kim WS, Choi YD, et al. Analysis of mutations in epidermal growth factor receptor gene in Korean patients with non-small cell lung cancer: summary of a nation- wide survey. J Pathol Transl Med 2015;49:481-488.

15. Carey KD, Garton AJ, Romero MS, et al. Kinetic analysis of epidermal growth factor receptor somatic mutant proteins shows increased sensitivity to the epidermal growth factor receptor tyrosine kinase inhibitor, erlotinib. Cancer Res 2006;66:8163-8171.

16. Jackman DM, Yeap BY, Sequist LV, et al. Exon 19 deletion mutations of epidermal growth factor receptor are associated with prolonged survival in non-small cell lung cancer patients treated with gefitinib or erlotinib. Clin Cancer Res 2006;12:3908-3914.

17. Riely GJ, Pao W, Pham D, et al. Clinical course of patients with non-small cell lung cancer and epidermal growth factor receptor exon 19 and exon 21 mutations treated with gefitinib or erlotinib. Clin Cancer Res 2006;12(3 Pt 1):839-844.

18. Sun JM, Won YW, Kim ST, et al. The different efficacy of gefitinib or erlotinib according to epidermal growth factor receptor exon 19 and exon 21 mutations in Korean non-small cell lung cancer patients. J Cancer Res Clin Oncol 2011;137:687-694.

19. Won YW, Han JY, Lee GK, et al. Comparison of clinical outcome of patients with non-small-cell lung cancer harbouring epidermal growth factor receptor exon 19 or exon 21 mutations. J Clin Pathol 2011;64:947-952.

20. Lee VH, Tin VP, Choy TS, et al. Association of exon 19 and 21 EGFR mutation patterns with treatment outcome after first-line tyrosine kinase inhibitor in metastatic nonsmall-cell lung cancer. J Thorac Oncol 2013;8:1148-1155.

21. Rosell R, Moran T, Queralt C, et al. Screening for epidermal growth factor receptor mutations in lung cancer. $\mathrm{N}$ Engl J Med 2009;361:958-967.

22. Kim DW, Lee SH, Lee JS, et al. A multicenter phase II study to evaluate the efficacy and safety of gefitinib as first-line treatment for Korean patients with advanced pulmonary adenocarcinoma harboring EGFR mutations. Lung Cancer 2011;71:65-69.

23. Igawa $S$, Kasajima M, Ishihara $M$, et al. Comparison of the efficacy of gefitinib in patients with non-small cell lung cancer according to the type of epidermal growth factor receptor mutation. Oncology 2014;87:215-223.

24. Asahina H, Yamazaki K, Kinoshita I, et al. A phase II trial of gefitinib as first-line therapy for advanced non-small cell lung cancer with epidermal growth factor receptor mutations. Br J Cancer 2006;95:998-1004.

25. Sequist LV, Martins RG, Spigel D, et al. First-line ge- 
fitinib in patients with advanced non-small-cell lung cancer harboring somatic EGFR mutations. J Clin Oncol 2008;26:2442-2449.

26. Sugio $\mathrm{K}$, Uramoto $\mathrm{H}$, Onitsuka $\mathrm{T}$, et al. Prospective phase II study of gefitinib in non-small cell lung cancer with epidermal growth factor receptor gene mutations. Lung Cancer 2009;64:314-318.

27. Inoue A, Suzuki T, Fukuhara T, et al. Prospective phase II study of gefitinib for chemotherapy-naive patients with advanced non-small-cell lung cancer with epidermal growth factor receptor gene mutations. J Clin Oncol 2006;24:3340-3346.

28. Wang H, Huang J, Yu X, et al. Different efficacy of EGFR tyrosine kinase inhibitors and prognosis in patients with subtypes of EGFR-mutated advanced non-small cell lung cancer: a meta-analysis. J Cancer Res Clin Oncol 2014;140:1901-1909.

29. Zhang Y, Sheng J, Kang S, et al. Patients with exon 19 deletion were associated with longer progression-free survival compared to those with L858R mutation after firstline EGFR-TKIs for advanced non-small cell lung cancer: a meta-analysis. PLoS One 2014;9:e107161.

30. Lee CK, Wu YL, Ding PN, et al. Impact of specific epidermal growth factor receptor (EGFR) mutations and clinical characteristics on outcomes after treatment with EGFR tyrosine kinase inhibitors versus chemotherapy in EGFR-mutant lung cancer: a meta-analysis. J Clin Oncol 2015:33:1958-1965.

31. Sheng M, Wang F, Zhao Y, et al. Comparison of clinical outcomes of patients with non-small-cell lung cancer harbouring epidermal growth factor receptor exon 19 or exon 21 mutations after tyrosine kinase inhibitors treatment: a meta-analysis. Eur J Clin Pharmacol 2016;72:1-11.

32. Choi YW, Jeon SY, Jeong GS, et al. EGFR exon 19 deletion is associated with favorable overall survival after first-line gefitinib therapy in advanced non-small cell lung cancer patients. Am J Clin Oncol 2016 Mar 10 [Epub]. http://doi. org/10.1097/COC.0000000000000282.

33. Mulloy R, Ferrand A, Kim Y, et al. Epidermal growth factor receptor mutants from human lung cancers exhibit enhanced catalytic activity and increased sensitivity to gefitinib. Cancer Res 2007;67:2325-2330.

34. Zhu JQ, Zhong WZ, Zhang GC, et al. Better survival with EGFR exon 19 than exon 21 mutations in gefitinib-treated non-small cell lung cancer patients is due to differential inhibition of downstream signals. Cancer Lett 2008;265:307-317.

35. Okabe T, Okamoto I, Tamura K, et al. Differential constitutive activation of the epidermal growth factor receptor in non-small cell lung cancer cells bearing EGFR gene mutation and amplification. Cancer Res 2007;67:20462053.

36. Okabayashi Y, Kido Y, Okutani T, Sugimoto Y, Sakaguchi K, Kasuga M. Tyrosines 1148 and 1173 of activated human epidermal growth factor receptors are binding sites of Shc in intact cells. J Biol Chem 1994;269:18674-18678.

37. Engelman JA, Zejnullahu K, Gale CM, et al. PFoo299804, an irreversible pan-ERBB inhibitor, is effective in lung cancer models with EGFR and ERBB2 mutations that are resistant to gefitinib. Cancer Res 2007;67:11924-11932.

38. Furuyama K, Harada T, Iwama E, et al. Sensitivity and kinase activity of epidermal growth factor receptor (EGFR) exon 19 and others to EGFR-tyrosine kinase inhibitors. Cancer Sci 2013;104:584-589.

39. Chen LY, Molina-Vila MA, Ruan SY, et al. Coexistence of EGFR T790M mutation and common activating mutations in pretreatment non-small cell lung cancer: a systematic review and meta-analysis. Lung Cancer 2016;94:46-53. 